\title{
Macro Photography with a Digital Camera
}

\author{
Vasily Yarovenko \\ Doctor of law, Professor, Law school, Far Eastern Federal University, the Honored of law the Russian Federation \\ Vladivostok, Russia; Email: yarovenko.vv@dvfu.ru
}

Tatyana Yarovenko

PhD. in Law, Ass. Professor, expert-criminalst

\section{Doi:10.5901/mjss.2015.v6n5s2p36}

\section{Abstract}

\begin{abstract}
Macro photography - it is a method of obtaining photographic images of objects on a large scale. Belarus photo is an intermediary between simple and macro photography, it is equal to 1:10 of 20:1. Rules and recommendations developed by the macro photography are also used in combination with other methods. The special advantage is the possibility to manufacture photographic macro photography high magnification of separate sites of filming, significantly larger than field of view microscope (Belkin, Korukhov, 1992). The challenge for macro focus consists in the making of images in a given scale with a clear transfer of the most important characteristics of investigated objects. It is on the scale of the imagery in most cases is a picture of the object, poorly conceived or even completely invisible to the naked eye in nature (Silkin, 1979). Object classification, a macro: - the material from which they are composed, opaque and transparent; - the degree of spatial properties-volumetric or flat, with a smooth or embossed; - the ability of a surface to reflect light-frosted with diffuse reflection and glossy with a mirror image of the rays. Quality images in macro photography are possible with proper identification needed exposure, accurate information on sharpness, as well as the location of the light source. Where, to get images with high quality under conditions of macro photography, in addition to the above conditions, you need to choose the right equipment for photographing objects.
\end{abstract}

Keywords: macro photography, digital camera, lens, flash, finger marks, exposure time, aperture

\section{Introduction}

In the criminalistical literature and practice focuses on photographing the general appearance of the scene environment and individual objects. However, it does not provide effective recommendations for photography using digital cameras individual parts and elements for better fixation of important information On the basis of the conducted experiments sponsored recommendations for practitioners on the use of modern digital cameras. Digital technologies can greatly simplify the process of fixing and increase manufacturing quality photos.

\section{Methodology}

The study was conducted from 2010-2015 years. The authors analyzed the theoretical and empirical research, which were made in previous years by other authors. Based on analysis and experiments have developed effective ways macro objects and different tracks as in situ and in the laboratory.

\section{Results}

One of the advantages of digital technology is that many digital cameras have a macro mode. In traditional photography, the term "macro" refers to lenses, vosproizvodivshie on 35-mm negative of the image in full size. In digital photography, there is no such thing as "negative of standard size, so all changes at the microscopic level are meaningless. The only important thing is to determine: whether the minimum focusing distance sufficiently close to an object (King, 2005). The resulting digital images if necessary can be increased by using a photo editor or during printing (photo 1, 2, 3). 

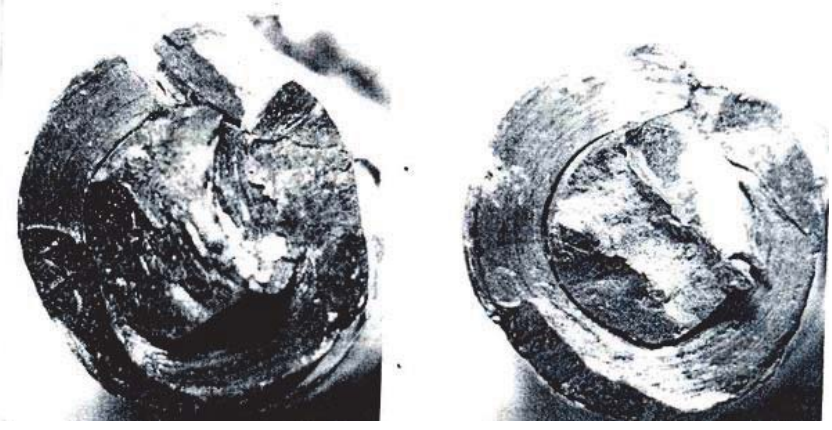

Photo 1, 2. General view of the cut marks on the end the surfaces of the ear lock

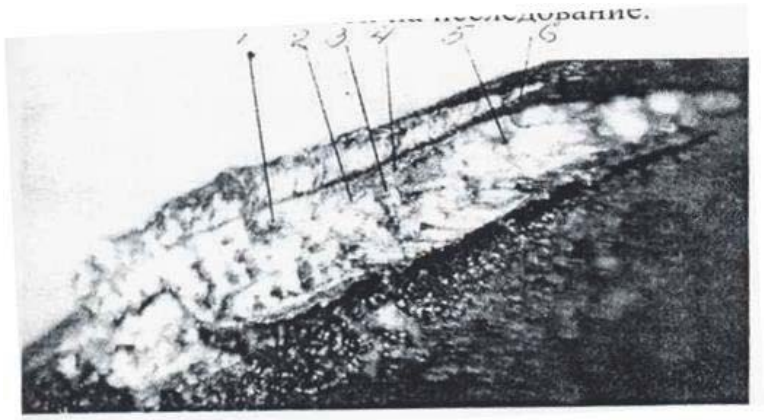

Photo 3. Larger image of the pilot footprint

If the increase in digital camera for any group of traces does not suffice, in this case, you must use special lenses that give a great increase that is macro lens. The important advantage of macro lenses are that no exposure compensation. With such a lens can produce shooting small objects in the same way as ordinary objects. Imagery during the inspection of the scene by making the makrosnimok papillary pattern, you can switch to shooting various objects without using special devices (photo 4, 5).

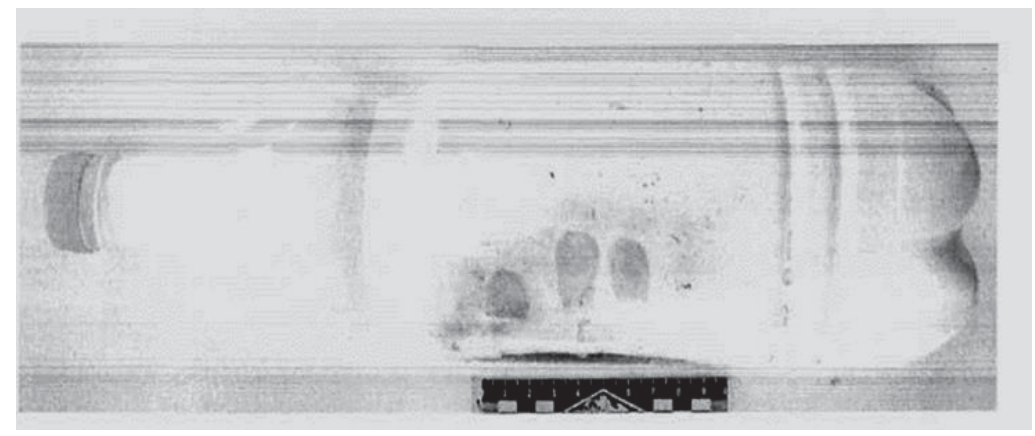

Photo 4. General view of bottle and papillary 


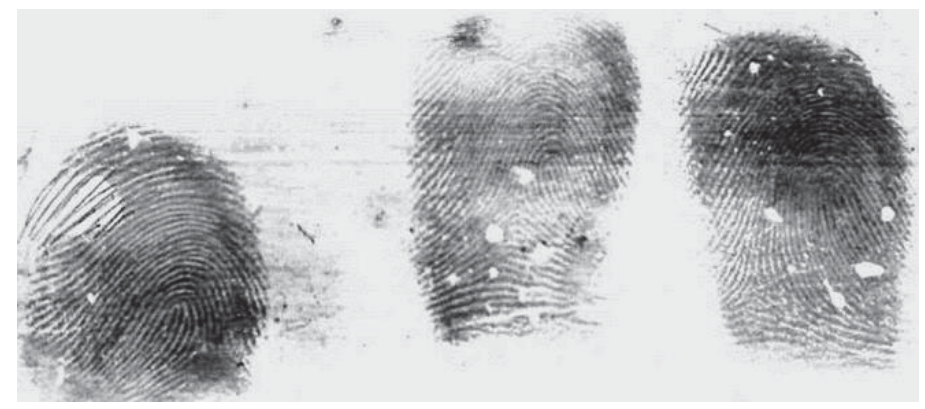

Photo 5. The increased traces of hands

As an example, look at the company's production Canon, which has developed various versatile lens for macro photography, to include expert research, we recommend the following:

1. EF $50 \mathrm{~mm} \mathrm{f} / 2.5$ Compact Macro

Minimum focusing distance $23 \mathrm{~cm}$ wide 1 shot: 1 . focal length $50 \mathrm{~mm}$ aperture ratio 1: 2.5; minimum aperture: 32; field of view angle - 46 degrees; the light filter diameter - $52 \mathrm{~mm}$ weight: $280 \mathrm{~g}$. (photo 6).

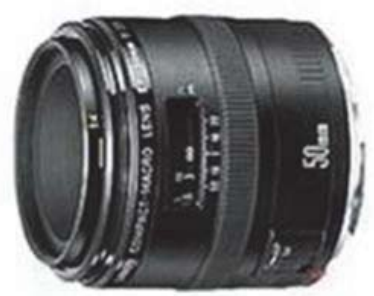

Photo 6. EF $50 \mathrm{~mm} \mathrm{f} / 2.5$ Compact Macro

2. Sigma EF70 - 300/4 - 5.6 New Macro, the Camera lens EF70-300. Macro language., one element of nizkodispersnogo glass; focal length - 70-300 mm; lens aperture 4-5,6; diaphragm max 4-5,6; diaphragm min 22-32; filter diameter $58 \mathrm{~mm}$; lenses/groups 14/10; telezum; MDF: 1.5 (0.95) m. size 74, 5x119, 5 mm; weight - 585 g. (photo 7).

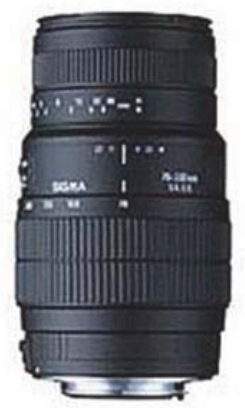

Photo 7. Sigma EF70 - 300/4 - 5.6 New Macro 
3. Sigma EF28 - 135/3.8 - 5.6 IF Macro, camera lens EF 28-135 focal length - 28-135 mm; lens aperture 3.8-5.6; diaphragm max- 3.8-5.6; diaphragm min2-32; filter diameter $62 \mathrm{~mm}$; lenses/groups 13/12; telezum; MDF: $0.24 \mathrm{~m}$. size $75 \times 75 \mathrm{~mm}$;. Weight - $410 \mathrm{~g}$. (photo 8).

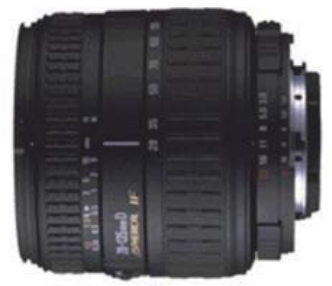

Photo 8. Sigma EF28 - 135/3.8 - 5.6 IF Macro

4. EF $100 \mathrm{~mm} \mathrm{f/2} \mathrm{USM} \mathrm{macro} \mathrm{ultrasonic} \mathrm{motor;} \mathrm{focal} \mathrm{length:} 100 \mathrm{~mm}$; lens aperture 2.8 diaphragm max - 2,8; diaphragm min -32; lenses/groups 12/8; MDF: $0.31 \mathrm{~m}$. size 75×105,3 mm; weight - $600 \mathrm{~g}$. (photo 9).

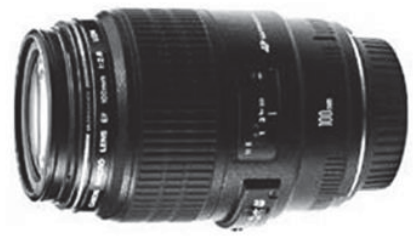

\section{Photo 9. Model EF 100 mm f/2 USM Macro}

Among lenses manufactured by other firms, consider the lens for macro Photography Company Olympus. Macro lens ZUIKO DIGITAL ED $50 \mathrm{~mm}$ f/2.0. In the lens used lenses from special nizkodispersionnogo (ED) glass. Resulting from the use of this lens images are bright and saturated. Focal length is $50 \mathrm{~mm}$; minimum focusing distance -0.24; aperture-1: 2.0; diaphragm min 22; number of petals -7; construction (elements/groups) - 11/10; field of view angle - 24 degrees; diameter filter $52 \mathrm{~mm}$; type lens hood LH-55; size - 71x61,5; weight - $300 \mathrm{~g}$. (photo 10).

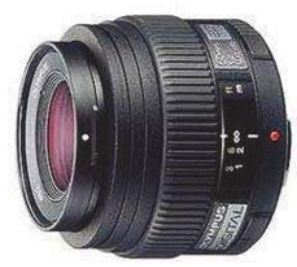

Photo 10. Zviko digital ED $50 \mathrm{~mm}$ f/2.0.

As demonstrated by testing more often, the experts at the disposal there are only compact digital cameras, but any additional equipment (adaptors, converters) to them. The drawback of such cameras with macro photography is that they have poor vision and no thread for mounting the macro lenses. Design of a compact camera, however, allows the use of special conversion or corrective lenses.

Converters to reduce light output, but increase the object. Choose the conversion factor is $1.4 \mathrm{x}$. It should be 
borne in mind that installing any lens on a digital camera with smaller sensor itself gives increase the magnification by a crop-factor, so is the case with an additional mounting converter this value grows even more: for example, 1.4x1.5=2.1.

Consider conversion applications, developed by Canon:

- teleconvertor TC-DC52A with a $52 \mathrm{~mm}$ thread is suitable for shooting high magnification (telephoto) Converter changes the focal length of the camera lens in 1.75, allows lens hood or filter installation (photo 11).

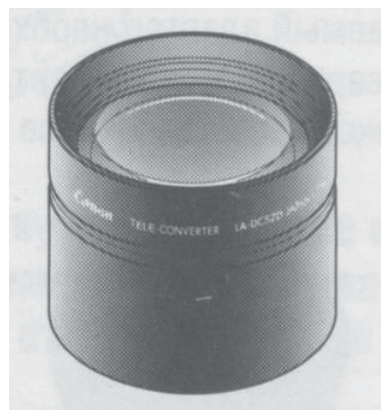

\section{Photo 11. Teleconvertor TC-DC52A}

- makrokonvertor 250D $(52 \mathrm{~mm})$ is designed for shooting from close range when the mode is set in the camera "macro", this converter allows you to shoot targets at a distance of 8 to $11 \mathrm{~cm}$ from the end to the lens (photo 12).

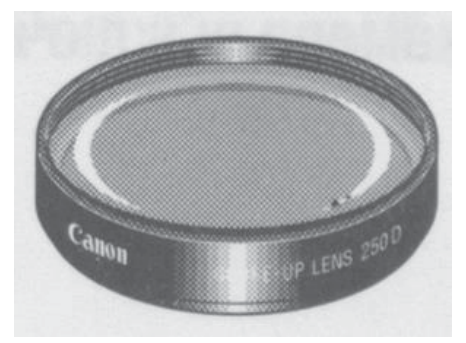

Photo 12. Makrokonvertor 250D (52 MM).

Using the above conversion to digital camera, perhaps if there are converters, for example, the adapter the adapter firm Canon LD-DC52(D) $(52 \mathrm{~mm})$ in macro mode for each digital apparatus is described in the instructions supplied with the digital camera (photo 13).

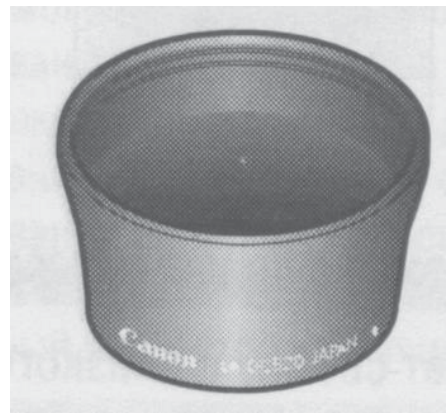

Photo 13. Adapter firm Canon LD-DC52D (52 мm.) 
Additionally we recommend to use corrective lenses for macro photography, lenses, manufactured by the company Tiffen. The optical power of the lens, as conventional, is measured in diopters and sets the numbers $+1,+2$, And so the higher the number, the stronger the lens increases and the less the distance at which you can focus it. If you put a few collecting lenses on top of each other, the power increase of such "pile" would be the sum of the components of the kit lens forces (King, 2005). Moreover it should be borne in mind that to use simultaneously more than 2 lenses is not recommended, since it increases distortions which adversely affect the clarity of the image. Close up lenses for macro can have different optical power or magnification. They provide an acceptable image quality, but not like that provide shooting lenses designed specifically for macro photography.

With macro photography a corrective lenses, consider what is better is in the centre of focus than at the edges, as the center image is clearer. Be aware that reducing the focal length and the aperture increases the depth of field also makes clear that most of the photos. Lenses with optional switching camera in the macro mode, but this usually gives the opportunity to push the camera closer to the object while keeping the same capture sharpening (King, 2005).

However, macro photography is required:

1. The extension rings. They navinchivautsya between the camera and the lens, resulting in improved ability to close-ups when selecting rings must take into account the fact that not all models provide preservation of health of the automated features of the lens, but only the most expensive model rings.

2. The Return of the ring. Navinchivautsya on the front part of a standard or wide angle lens, and the other party are joined to the bayonet mount to the camera for $24 \mathrm{~mm}$ wide angle lens bellows in such a combination extension receive a six fold increase.

3. The connecting rings. Enable you to collect lenses navinchivya. Lens attached to the camera, fully preserves electronic controls. The second lens will have to be operated manually. Magnification ratio is calculated as the ratio of the first and second focal lengths of lenses.

- $\quad$ Nozzles with bellows. They can becombined with makroob"ektivami, reverse rings, mikroskopnymi lenses, etc.

One of the problems of macro photography is a lighting object. In macro photography, we recommend positioning the light source before photographs, so not to create a shadow on the subject. Furthermore, you must disable the built in flash, as part of the photo will be bright. If the built in flash is the only source of illumination, the lighting can be reduced, having covered with a piece of white plastic or thin white cloth. However, as a general rule, macro photography on commit objects, you must use makrovspyski.

For example, the flash at full power Macro Twin-Light the firm Canon. This flash consists of two modules that attach to the camera with a special ring adapter. The proposed design allows tilt modules as well as their movement around the lens and the ability to adjust the charge for each module individually. Design allows tilt of modules and also enables you to move the modules around the lens, for optimal illumination of the object during filming. Another feature of this outbreak is that possible to adjust the charge, with each module separately (photo 14).

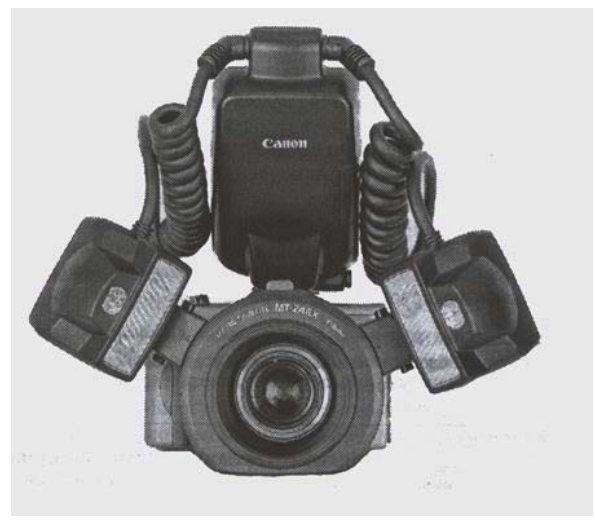

Photo 14. Flash for macro photography

Let's take one more photo flash for macro photography, called "ring". «Ring» flash is especially useful when photographing small objects. The main technologies used in the action principle of circular lighting (Sajiev, Yarovenko, 
1994, Tunis, 1998). It put on the camera lens, and it automatically triggered when photographing with a built in flash (photo 15).

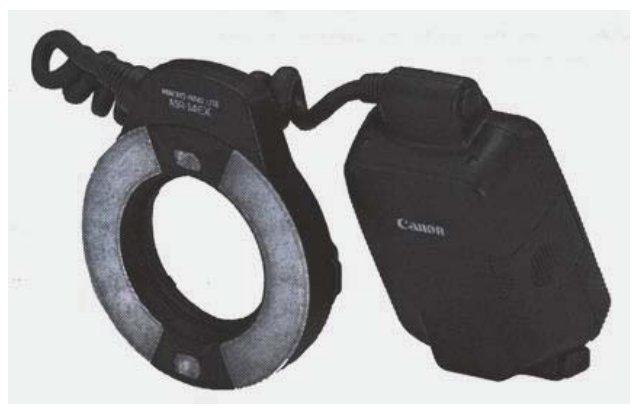

Photo 15. Ring Flash

Depending on how the object is the result of increased image quality. Look at conditions on the image quality:

1. View angle. If you increase the focal length becomes narrower angle of view of the camera. Image taken from afar with a larger focal length, will contain fewer parts than a motion background with smaller focal length, but is located close to the object.

2. Spatial correlation. Moving the camera also changes the relative position and size of your subject relative to background objects.

3. Increase the focal length reduces the depth of field, which affects the quality of the snapshot.

Therefore, in situations macro depth of field is important, and understanding it helps achieve significant results. Depth of field is the place where the lens is focused on an object, light rays reflected from it, are projected into the focal plane as points of light. The focal plane and these rays form an did not point, and mugs. The diameter of such "circles" scattering increases with increasing distance from the image plane, the image itself is becoming increasingly blurred as the resolving power of the eye is limited, there is a certain threshold diameter circles of dispersion, they are perceived by the eye as a point-it is on the basis of this fact and works the notion of depth of field (Thomson, (2005).

By law, any lens optics ideal will focus in its focal plane of only those objects that are in strictly certain distance from it. Rays of light reflected from an object lying in that plane, projected onto the camera matrix in the form of subtle lighting points. The other rays reflected from the lens, behind or in front of the plane, form no longer points, and different diameter circles, called "circles of dispersion. They increase in size as the distance between the objects and their corresponding plane of perfect focus so that objects in the picture are becoming more blurred. And since the human eye has limited resolution, around the plane of perfect focus there is always some space where the image formed is not "perfectly sharp points and circles, which are seen scattering eye also sharp as points. This zone is called sharply represented space (RIP), and its depth-depth of RIP or just the depth of field.

Maximum diameter of mug, which is perceived by scattering the human eye as a point, is called a "valid" scattering circle. The diameter depends on the size of the light sensitive matrix, image print size and the distance at which the image will be considered. Depth of field can range from millimeters to infinity. Depth of field is determined by three factors.

The first and main is the diaphragm. If your camera allows you to adjust the aperture, the more closed aperture the greater the depth of field, and vice versa (provided the focal length and subject distance remain unchanged).

The second factor is the focal length. When you increase the focal length of the RIP is significantly reduced due to the increase of image that becomes noticeable in the work with powerful telephoto lenses.

The third factor is the distance from the camera to the point of focus. To obtain high quality images must be experimentally choose the distance between the camera and the object.

Macro and telephoto lenses for depth of field is distributed at the same distance behind and in front of the plane of focus. Some digital Canon there are modes DEP or A-DEP for choosing near and far points of the frame to be in focus.

As an alternative, you can use the depth of field table attached to the lens. This table shows the distance to the front and rear of the border RIP with different values of aperture and varying the distance of the subject. Macro lenses have this scale expanded and provide greater accuracy, while the scale of the optics is starting to count down from the meter and have less precision. 
It should be noted that table depths of field and appropriate scale of lenses usually are calculated under a film camera rather than under digital cameras with light sensitive matrix size in most cases is less than the standard 35-mm square negativity. Because of this disparity arises and squares the difference in diameter permissible of circles-for film cameras scattering is considered equal to $0.025 \mathrm{~mm}$, while the digital camera must be reduced to about $0.02 \mathrm{~mm}$. Reducing the diameters of the circles have a scattering of digital cameras reduces the depth of field compared with film counterparts at 30-60\% (all other things being equal: the same focal length, aperture and subject distance).

Compact digital cameras have even smaller matrices than digital looking-glass cameras. So, in order to ensure comparable angles, you must apply the korotkofokusnuy optics (such, with real lengths 5-15 mm).

4. Exposition. When changing the focal length you must select the shutter speed and aperture. Auto exposure mode the camera performs this setting yourself. In manual mode, all changes make taking pictures.

Currently determining the correct exposure of macro is missing. It is determined experimentally. The difficulty is that in a picture taking over of field with small distances increases the conjugate focal distance and existing aperture remains unchanged.

5. Distortion. When shooting at wide angle lens, you can face the problem of distortion. This is due the fact that vertical lines located on the edges of the photos, start to wind around the inside of the frame. It should be borne in mind that shooting wide angle mode object at close range distorts proportions (photo 16,17 ).
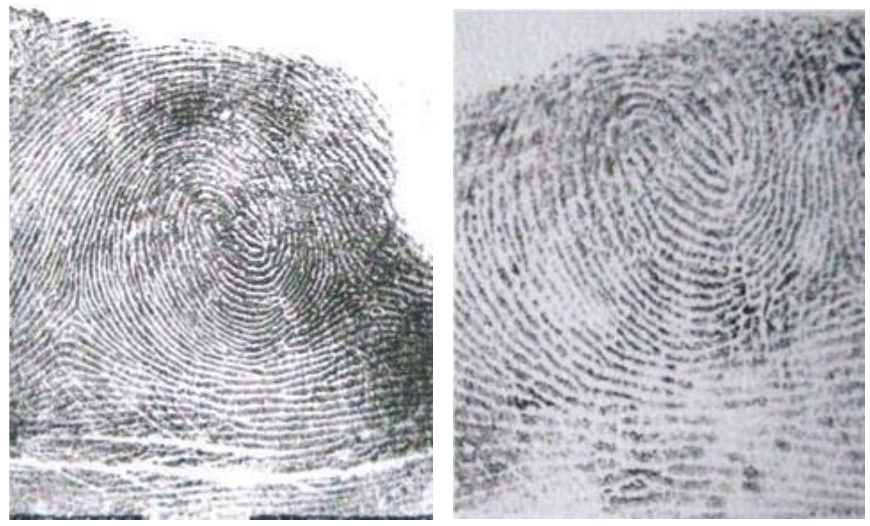

Photo 16,17 . The trail of the same finger on two fingerprint cards with distortion

Correct lens has great importance in macro photography.

1. Variable focal lenses don't always allow you to use the full range of optical magnification. Normally the LCD screen appears, indicating that the focal length is allowed for macro mode or out of them.

2. Measuring the focal length also affects the minimum allowable distance.

3. With good lighting an object is in the process of focusing and more qualitatively higher quality photos.

4. There are lenses that have the most sharp images are obtained with the average height of the diaphragm. But the depth of field is less than the minimum aperture of the lens, i.e. the narrow bounds of the area that the shot will turn out well.

5. Problems with sharpness is not only improper, but also because of the bias of the camera when taking any slightest camera shake affects the quality snapshot. In this case, the macro, you must use a tripod.

If filming is done on the camera, the viewfinder is not associated with lens, (these cameras usually include compact cameras), the layout of the frame in macro to use the LCD screen preview. This is due to the fact that the viewfinder lens effect is not visible. While photographing when using liquid crystalline viewfinder doesn't always give a positive result. This refers to the moment when photography takes place in low light conditions.

At the same time, professional cameras, the optical viewfinder is associated with lens, that is identical to synchronized and show the same image, but in any case as a control to access the preview screen.

There is another way to macro zoom function: Marco. So, when shooting in wide angle lens when marco gives a clear image as the object and the background. When shooting at telephoto image object is allocated on a blurred background. This is because in cases where the lens is at the maximum wide angle, the amount withdrawn is 
approximately 56x42 $\mathrm{mm}$ (maximum wide angle position), and when the lens is set to telephoto-87x65 mm (maximum lens length (telephoto). When the zoom-lens is in the intermediate position between the distance from the lens to the object equals the distance in telephoto.

In addition, digital zoom in digital cameras sold mode optical zoom. Optical zoom is performed using variable focal lenses. When the digital zoom shooting frame first, and then the camera increases the size of the image in pixels and trims out beyond the frame pieces. A similar result can be obtained by using a graphical editor. But both the first and the second case is the quality of the pictures: it is lower than is possible when taking a zoom lens. This is because filling the gaps between the pixels of an image that a computer system of the camera adds new pixels, that is recalculating the shot. The process of inserting pixels between existing always has a negative influence on the development of small parts of the image. It should be noted that there are models of digital cameras, which do not translate their image, saving it with fewer pixels.

In addition, adjusting lens focal length is accompanied by a change in the angle of view and depth of field, while the digital zoom settings remain constant as the focal length of the lens does not change. Digital zoom also has no effect on the exposure, geometric distortion and spatial relationship between objects. Mainly when shooting close up, you must use the maximum resolution and maximum compression (photo 18, 19).

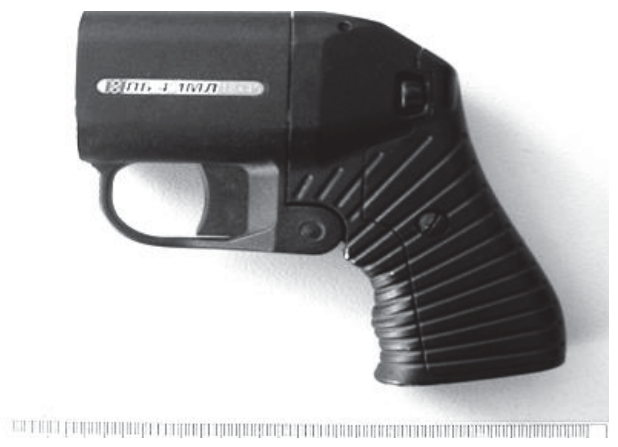

Photo 18. Pistol №. K 016059 submitted to on study

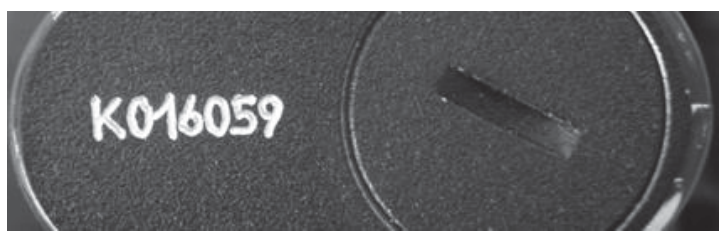

Photo 19. The serial number on the bottom of the Pistol grip handle

Currently in expert centers in macro photography instead of cameras use a scanner, but this technique is, in our view, does not apply to macro photography - is one of the ways to obtain reproductions.

\section{Conclusion}

As a result of the use of digital devices and equipment greatly enhanced opportunities to use macro on fixing various marks on objects. Considered ways are simple and accessible to practitioners. They do not require special education and expensive equipment, because the modern digital cameras have a macro mode.

\section{References}

Belkin, R.S., Korukhov U.Ya. 1992. Fundamentals of Criminalistics. Moscow: Progress Publishes, pp. 168-170.

King, D. 2005. On digital photography: become a professional! Self study course. Piter, p. 113.

Sajfiev, R.I., Yarovenko, V.V. 1994. Theory and practice of improving detection tools and fixing invisible palm prints. Vladivostok: 
Dal'nevost. university press, p. 122.

Silkin, P.F. 1979. Forensic research picture. Volgograd: Higher school of the USSR MINISTRY OF INTERNAL AFFAIRS, p. 56. Thomson, A. 2005. Master of digital camera. Journal of DigitalPhoto, 2005, № 7, p. 86.

Tunis, I.O. 1998. Acting photographic fixing the traces of fingers using UFL. Vladivostok.Far eastern forensic read, p. 40. 